\section{Utility of endoscopic ultrasound in idiopathic acute recurrent pancreatitis}

To cite: Tepox-Padrón A, Bernal-Mendez RA, DuarteMedrano G, et al. Utility of endoscopic ultrasound in idiopathic acute recurrent pancreatitis. BMJ Open Gastro 2021;8:e000538. doi:10.1136/ bmjgast-2020-000538

Received 7 September 2020 Revised 23 November 2020 Accepted 24 November 2020

Check for updates

C C Author(s) (or their employer(s)) 2021. Re-use permitted under CC BY-NC. No commercial re-use. See rights and permissions. Published by BMJ.

${ }^{1}$ Gastrointestinal Endoscopy Department, Instituto Nacional de Ciencias Medicas y Nutricion Salvador Zubiran, Mexico,

Mexico

${ }^{2}$ Gastroenterology Department, Instituto Nacional de Ciencias Medicas y Nutricion Salvador Zubiran, Tlalpan, Mexico

${ }^{3}$ Surgery Department, Instituto Nacional de Ciencias Medicas y Nutricion Salvador Zubiran, Mexico, Mexico

Correspondence to Dr Felix Tellez-Avila; felixtelleza@gmail.com

\section{ABSTRACT:}

Idiopathic acute recurrent pancreatitis (IARP) is defined as at least two episodes of acute pancreatitis with the complete or near-complete resolution of symptoms and signs of pancreatitis between episodes, without an identified cause. There is a paucity of information about the usefulness of endoscopic ultrasound (EUS) in IARP. Objectives To determine the diagnostic yield of EUS in IARP.

Design A retrospective study was performed in patients with IARP evaluated by EUS between January 2009 and December 2016. Follow-up assessments of acute pancreatitis recurrence were carried out.

Results Seventy-three patients with 102 EUS procedures were included. EUS was able to identify the cause of IARP in 55 patients (75.3\%). The most common findings were chronic pancreatitis in 27 patients (49.1\%), followed by lithiasic pathology in 24 patients (43.6\%), and intraductal papillary mucinous neoplasm in four patients (7.3\%). A directed treatment against EUS findings had a protective tendency associated with the final resolution of recurrence. There were no complications reported. Conclusion EUS performed in patients with IARP helped to identify a possible cause in $2 / 3$ of the cases. The majority of patients have a treatable disease.

\section{INTRODUCTION}

Acute pancreatitis (AP) is the most common disease affecting the pancreas and has a high incidence worldwide. ${ }^{1}$ After the first attack of AP, $22 \%$ develop at least one episode of recurrence and $10 \%$ develop chronic pancreatitis (CP). ${ }^{12}$ Risk factors for the transition to recurrent and $\mathrm{CP}$ is related to the cause of $\mathrm{AP}^{3}$ However, the cause is not always obvious, despite the complete evaluation of history, laboratory and imaging. Approximately $10 \%-30 \%$ of patients with AP are in a category labelled as idiopathic AP (IAP). ${ }^{24-7}$ This group is prone to a high recurrence rate that can reach up to $70 \% .8$ Acute recurrent pancreatitis is defined as at least two episodes of AP with the complete or near-complete resolution of symptoms and signs of pancreatitis between episodes. ${ }^{26}$ In this scenario, a

\section{Summary box}

What is already known about this subject?

- Idiopathic acute recurrent pancreatitis (IARP) is defined as at least two episodes of acute pancreatitis with the complete or near-complete resolution of symptoms and signs of pancreatitis between episodes, without an identified cause. There is a paucity of information about the usefulness of endoscopic ultrasound (EUS) in IARP.

What are the new findings?

- EUS performed in patients with IARP helped to identify a possible cause in $2 / 3$ of the cases, with chronic pancreatitis and biliary lithiasis pathology being the most frequent findings.

How might it impact on clinical practice in the foreseeable future?

- Performing EUS in patients with IARP can help to identify and treat the cause, avoiding recurrence in most cases.

more extensive assessment including endoscopic retrograde cholangiography (ERCP), endoscopic ultrasound (EUS) or magnetic resonance cholangiopancreatography (MRCP) is used to determine the aetiology. ${ }^{7}$

EUS has been reported to be the imaging of choice for the pancreas and biliary tract. EUS is a safe, minimally invasive diagnostic procedure in patients with idiopathic acute recurrent pancreatitis (IARP). The diagnostic yield of EUS might be up to $80 \%$ of patients. ${ }^{89}$ The aim of this study was to evaluate the diagnostic yield of EUS in patients with IARP.

\section{METHODS}

A retrospective study based on a prospective data collection was conducted at a single tertiary institution from January 2009 to December 2016. The information was obtained from endoscopic reports as well as 
physical and electronic health records from patients diagnosed with IARP. All patients underwent EUS examination. Exclusion criteria included the following: patients aged under 15 years; medical contraindication for EUS; patients referred from another institution; a documented ongoing alcohol and tobacco consumption; and metabolic conditions known to predispose to pancreatitis (including hyperparathyroidism, hypercalcaemia and hypertriglyceridaemia of more than $500 \mathrm{mg} / \mathrm{dL}$ ).

In our centre, all patients with pancreatic diseases are evaluated, followed and treated by the Pancreas Clinic, as formed by gastroenterologists, pancreatologists, endoscopists and surgeons with a special interest in pancreatic diseases. Demographic data (age, sex, weight and height), prior surgery, previous AP and comorbidity were collected. Preprocedural laboratory tests were run on the entire cohort in order to assess the security of the procedure and the risk of bleeding. Diagnostic indications and the results of imaging techniques and EUS data were obtained.

EUS examinations were performed by two endoscopic sonologists with experience of more than 3000 EUS procedures. All patients underwent standard monitoring with a continuous display of the heart rate and pulse oximetry along with blood pressure. EUS procedures were performed under conscious sedation provided by an anaesthesiologist assisted by trained nurses. A linear echoendoscope GF-UCT140 (Olympus, Tokyo, Japan) with an Aloka ultrasound machine SSD-5500 (Aloka, Tokyo, Japan) and a linear echoendoscope FUJI (eg, -530UT) with an ultrasound machine SU-8000 (Fujifilm, Minato-Ku, Tokyo, Japan) were used. Patients were hospitalised and underwent strict supervision for at least 2 hours after the procedure. Informed consent was obtained from all patients prior to the endoscopic procedure.

\section{Definitions}

The Atlanta classification 2012 was used to define AP. The diagnosis requires two of the following three features: (1) abdominal pain consistent with AP; (2) serum lipase or amylase activity at least three times greater than the upper limit of normal and (3) characteristic findings of AP on imaging (contrast-enhanced CT (CECT), MRI or transabdominal ultrasonography). ${ }^{10}$ IARP is defined as a condition in which at least two well-documented episodes of AP have occurred, with the resolution of symptoms between each episode. It is characterised by an absence of morphological criteria for CP by MRI or CECT and the absence of a specific diagnosis following clinical history and physical examination; laboratory data including triglyceride and calcium concentrations; a review of all medications, both prescription and over-the-counter and cross-sectional abdominal imaging studies including abdominal ultrasound for all patients with a gallbladder in situ. ${ }^{5}$ Severity was defined by the Atlanta classification 2012. ${ }^{10}$ We considered EUS findings to be diagnostic if they could explain RAP. Biliary aetiology was considered as follows: microlithiasis was defined as a hyperechoic signal of $0.5-3 \mathrm{~mm}$ without a postacoustic shadow. Biliary sludge was defined as moving echoes of low amplitude in the lumen of the gallbladder without any postacoustic shadow. Gallstones were defined as moving echoes of high amplitude of more than $3 \mathrm{~mm}$, with a postacoustic shadow. These findings could be present inside the gallbladder or common bile duct. ${ }^{11}$ The treatment of patients was tailored according to aetiology and was recorded as a surgical procedure (cholecystectomy) or endoscopic procedure (ERCP with sphincterotomy or stone extraction). CP was diagnosed according to the conventional criteria if five or more criteria were found; all identified endosonographic features were recorded. ${ }^{12-14}$ Pancreas divisum (PD) was diagnosed by EUS when there was clear evidence of a dominant dorsal duct with no evidence of communication between the ventral and dorsal ducts, ${ }^{8}$ with the ventral pancreatic duct coursing to the duodenal wall proximal and anterior to the bile duct, in the expected location of the minor papilla, ${ }^{15}$ or if the main pancreatic duct could not be traced from the major papilla. ${ }^{8}$ The diagnosis of autoimmune pancreatitis was established based on International Consensus Diagnostic Criteria. $^{16}$

After the EUS procedure, patients were followed up with clinical evaluation and recording of the presence of new episodes of AP. The last outpatient visit or inpatient stay was considered in the follow-up visitation.

\section{Statistical methods}

Demographic and clinical data were analysed using descriptive statistics, including absolute and relative frequencies. Median and minimum-maximum ranges were used in the nonparametric statistical analysis. We evaluated the association of the non-development of a new event of AP after treatment using the ORs. We calculated the OR as the likelihood that a new event of AP would occur given that the patient was undergoing treatment, compared with the odds of the outcome occurring in the absence of treatment. All analyses were performed using SPSS V.20.

\section{RESULTS}

Initially, the clinical records of a total of 101 patients were evaluated; 28 patients were excluded (figure 1). Seventythree patients were included. In $55(75.3 \%)$ patients, EUS findings were considered as diagnostic and in 18 (24.7\%), they were non-diagnostic. Thirty-seven $(67.3 \%)$ patients were male. The median age of the patients was 31 (range 15-75) years. The median of documented attacks of AP prior to EUS evaluation was four (range 2-13). Thirty-six $(49.3 \%)$ patients had a history of at least one episode of severe pancreatitis. Thirty-four $(46.6 \%)$ patients had a cholecystectomy before EUS evaluation; $88.2 \%$ of these patients were suspected of having a biliary cause of AP as an indication for surgery. All patients underwent at least 


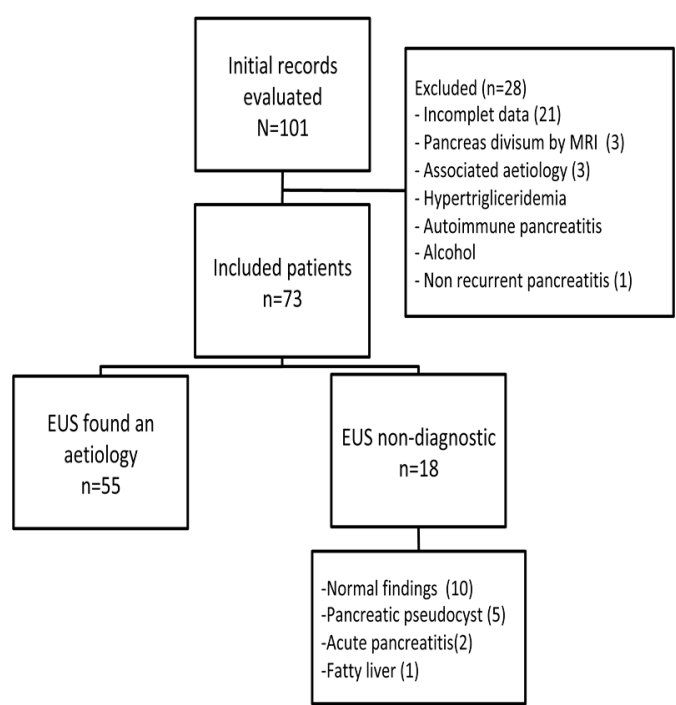

Figure 1 Flow chart of the patients included. EUS, endoscopic ultrasound.

one transabdominal ultrasonography and an abdominal CECT. MRCP was done in 20 patients prior to EUS.

An IARP aetiology was found in $55(75.3 \%)$ patients (table 1). The most common finding was $\mathrm{CP}(\mathrm{n}=27 / 55$; $49.1 \%$ ); ten patients had 3-4 criteria considered as early $\mathrm{CP}$ and $17 \mathrm{had} \geq 5$ criteria. Ten patients had a history of severe AP and biliary lithiasis was found in 24 (43.6\%) patients. Intraductal papillary mucinous neoplasm was found in $4(7.3 \%)$ patients: two had main duct IPMN, one patient underwent distal pancreatectomy and one patient refused surgery. One $5 \mathrm{~mm}$ branch-duct IPMN was in the tail of a patient who underwent distal pancreatectomy and one $20 \mathrm{~mm}$ mixed-type IPMN was reported in a patient who refused surgery; neither suffered recurrences at follow-up. In 28/55 (50.9\%) patients, a treatable cause was identified using EUS, of which $24(85.7 \%)$ underwent definitive treatment (ERCP/surgery). Four patients $(14.3 \%)$ refused the proposed treatment.

\begin{tabular}{lll}
\hline $\begin{array}{l}\text { Table } 1 \\
\text { EUS in included patients }\end{array}$ & \multicolumn{3}{l}{ Aetogies of acute pancreatitis documented by } \\
\hline Diagnosis & $\mathbf{n = 5 5}$ & \% \\
\hline Chronic pancreatitis & 27 & 49.1 \\
$\begin{array}{l}\text { Lithiasis pathologies } \\
\quad \begin{array}{l}\text { Gallbladder stones/sludge/ } \\
\text { microlithiasis }\end{array}\end{array}$ & 24 & 43.6 \\
$\begin{array}{l}\text { Choledochal lithiasis/sludge/ } \\
\text { microlithiasis }\end{array}$ & $4 / 1 / 1$ & - \\
$\begin{array}{l}\text { Intraductal papillary mucinous } \\
\text { neoplasm }\end{array}$ & 4 & 7.3 \\
$\quad \begin{array}{l}\text { Main duct } \\
\text { Branch-duct }\end{array}$ & 2 & - \\
$\quad$ Mixed type & 1 & - \\
\hline
\end{tabular}

EUS, endoscopic ultrasound.
Table 2 Recurrence rate of pancreatitis in patients with treatable diseases detected by EUS classified by following treatment and without treatment according to EUS findings

\begin{tabular}{lll}
$\begin{array}{l}\text { Patients with EUS } \\
\text { attributed cause, } \mathbf{n = 2 8} \\
\mathbf{n}(\%)^{*}\end{array}$ & $\begin{array}{l}\text { With recurrence } \\
\mathbf{n}(\%)\end{array}$ & $\begin{array}{l}\text { Without } \\
\text { recurrence } \\
\mathbf{n}(\%)\end{array}$ \\
\hline $\begin{array}{l}\text { With treatment } \\
24(85.7)\end{array}$ & $3(12.5)$ & $21(87.5)$ \\
$\begin{array}{l}\text { Without treatment } \\
4(14.3)\end{array}$ & $2(50)$ & $2(50)$
\end{tabular}

*Only patients with 'treatable diseases' detected by EUS are included in this table: lithiasis pathologies and intraductal papillary mucinous neoplasm.

EUS, endoscopic ultrasound.

One hundred and two EUS examinations were performed in 73 patients. Twenty-two patients had two or more EUS (median 2, range 2-4). Seventeen were performed as part of a research protocol about the follow-up of 'early CP'; in three patients requiring pancreatic pseudocyst drainage, one was evaluated prior and during a live demo, and one patient required the drainage of a $2 \mathrm{~cm}$ hepatic abscess in the left hepatic lobe.

\section{Follow-Up data}

The median follow-up was 36 months (range 1-144 months) after EUS. The incidence of new episodes of AP was lower for patients who underwent treatment according to EUS findings (table 2). Focused treatment according to EUS findings had a tendency towards the decreased risk of further episodes of AP, OR 0.14 (95\% CI 0.01 to 1.43 ), with the number needed to treat (NNT) being 2.7 patients.

No complications associated with the EUS procedure were reported.

\section{DISCUSSION}

According to our data, EUS is a useful tool with high diagnostic accuracy in evaluating patients with IARP and the EUS-based management strategy appears to be associated with decreased recurrence rates. The initial evaluation failed to detect the cause in up to $30 \%$ of patients with acute recurrent pancreatitis. ${ }^{17}$ The EUS has been effective in providing useful information in such patients, including the identification of colelithiasis, microlithiasis and choledocholitiasis. ${ }^{18}$ This strategy might be used when other non-invasive modalities have failed to identify the aetiology, in addition to being a minimally invasive modality with high accuracy. ${ }^{719}$ However, there is a paucity of studies establishing the specific role of EUS in IARP.

In this study, the diagnostic yield of EUS in the current population was $75 \%$, which correlates with previous publications reporting diagnostic yields varying from $29 \%$ to $88 \% .{ }^{182021}$ In a recent meta-analysis by Umans $e t a l$, the overall diagnostic yield was $59 \%$, and the diagnostic yield of EUS was similar in the first episode compared 
with IARP ( $56 \%$ vs $52 \%){ }^{22}$ Biliary tract disease was the most common treatable aetiology, which is similar to that reported in other studies. ${ }^{1722-24}$ In a prospective study including 31 patients, ${ }^{17}$ intraductal ultrasound detected the possible cause of IARP in $41.9 \%$ of patients. Bile duct stones and sludge were detected as the most common cause. Pereira et $a l^{18}$ reported a systematic review in patients with IAP undergoing EUS, in which biliary aetiology (microlithiasis or choledocholitiasis) was found in $37 \%$ of cases.

IARP is also defined as the occurrence of two or more episodes of AP without concurrent clinical or imaging evidence suggestive of CP by MRI, CECT or transabdominal ultrasonography. ${ }^{9}$ In our study, 27 (49.1\%) patients were diagnosed with CP in spite of previous negative studies. It is well known that EUS can detect these kind of patients, which are sometimes considered to have 'early CP'. ${ }^{12-14}$ On the other hand, it is important to point out that up to $50 \%$ of untreated patients with IARP might develop CP. Yusoff $e t a l^{21}$ found CP to be significantly higher in patients with recurrent pancreatitis compared with those with a single episode $(74.1 \%$ vs $53 \%$; $\mathrm{p}=0.0009$ ). Therefore, the percentage of $\mathrm{CP}$ in our study should be interpreted with caution, as this could represent some uninvestigated aetiology from the onset that could lead to $\mathrm{CP}$, rather than an aetiology. ${ }^{25} 26$ Nevertheless, CP with dilated PD, stricture or stones may benefit from endoscopic or surgical treatment in order to reduce recurrent episodes of AP or $\mathrm{CP} .{ }^{18}$ Due to these difficulties, we decided not to include patients with $\mathrm{CP}$ as patients with 'treatable disease'.

There are some studies that have reported a decrease in the diagnosis of biliary disease in patients without gallbladder in situ. ${ }^{20}{ }^{21}$ Vila et $a l^{24}$ found that the presence of gallbladder in situ had a greater tendency to identify a cause for IAP. Although a history of cholecystectomy was present in $46.6 \%$ of our patients, biliary disease was the second most prevalent after CP. Umans et al performed a meta-analysis which showed a higher diagnostic yield in patients with gallbladder in situ compared with prior cholecystectomy (50\% vs 64\%). ${ }^{22}$ According to our results, endoscopic or surgical treatment based on EUS findings was useful against new episodes of AP. We recommend the correct identification of biliary pathology in all patients with IARP before endoscopic or surgical treatment. In a retrospective study comparing cholecystectomy vs follow-up in patients with IAP, the recurrence of AP was lower in cholecystectomy patients compared with follow-up ( $14 \%$ vs $43 \%$; $\mathrm{p}=0.003) .{ }^{27}$ Räty et a ${ }^{28}$ confirmed these findings in a randomised trial reporting a recurrence rate of $14 / 37$ in the follow-up arm vs $4 / 35$ in the cholecystectomy arm (OR 4.7, 95\% CI 1.4 to $1.6, \mathrm{p}=0.008$ ) with a median follow-up of 24 months. Regarding sphincterotomy, Kim $e t a l^{17}$ showed an absence of recurrence in $87.5 \%$ of patients with bile duct stones or sludge after sphincterotomy during a 36-month follow-up period. Sajith et $a l^{23}$ demonstrated that when treatment was tailored according to aetiology, $73 \%$ of patients with RAP remained asymptomatic during the follow-up. Finally, Umans et al found a tendency in favour of a decreased recurrence rate in patients with biliary disease diagnosed by EUS (risk ratio $0.71,95 \%$ CI 0.21 to 2.41 ). ${ }^{22}$

Wilcox et al found a $40 \%$ recurrence rate in a prospective cohort of 121 patients with IARP. However, the two most frequent findings in this study were sphincter of Oddi dysfunction (SOD) and PD, which were both associated with high recurrence rates $(53 \%$ and $68 \%$, respectively) ${ }^{26}$ It is possible that the differences between the recurrence rates in these studies and our results are derived from differences in the aetiologies found in each group of patients. Also, we do not have access to a manometer in our centre to evaluate SOD. Therefore, we consider that the cause of the pancreatitis is directly related to the risk of recurrence.

Our study has some limitations. The first is the retrospective nature of the study, followed by the number of patients. However, the strength of our study is the close follow-up used to evaluate and demonstrate the performance of endoscopic and surgical treatment based on EUS findings. The strength of this approach was demonstrated by the tendency to avoid new AP episodes in most of patients, with a small NNT. Further limitations of our study include the lack of endoscopic manometry of the sphincter of Oddi, as well as the lack of genetic tests for IARP, which are rarely performed in our country outside of clinical trials. However, these resources are unavailable in the majority of centres, including reference centres. In the most recent meta-analysis, they found high heterogeneity in diagnostic work-up before EUS. ${ }^{22} 29$ Finally, 102 EUS were performed in 73 patients; in four patients, the aetiology was found in the second procedure. Although EUS is a useful tool, as an operator-dependent tool and being subjective to interpretive error, the pathology could be overlooked. We have to mention that the data of the present study are important in the global data because our results exclusively include patients with IARP and all of these patients have undergone the EUS procedure; as a result, our paper is the fourth biggest sample reported to date.

In conclusion, EUS is a useful tool with high diagnostic accuracy in evaluating patients with IARP. An EUS-based management strategy appears to be associated with decreased recurrence rates.

Contributors Téllez-Ávila FI, Tepox-Padrón A Bernal-Mendez RA, and DuarteMedrano $\mathrm{G}$ designed the report; Téllez-Ávila FI and Ramírez-Luna M performed endoscopies; Tepox-Padrón A Bernal-Mendez RA, Duarte-Medrano G, and Marroquín-Reyes JD collected data; TéllezÁvila Fl, Duarte-Medrano G, BernalMendez RA, Romano-Munive AF, Mairena-Valle M, Valdovinos-Andraca F, Uscanga L, Chan C, Domínguez-Rosado I, and Ramírez-Luna M organized the report; and Téllez-Ávila FI, Tepox-Padrón A, Marroquín-Reyes JD, andDuarte-Medrano G, wrote the paper.

Funding The authors have not declared a specific grant for this research from any funding agency in the public, commercial or not-for-profit sectors.

Competing interests AT-P, RAB-M, GD-M, AFR-M, MM-V, MAR-L, JDM-R, LU,CC, ID-R, JHC, MP-L and FT-A. FT-A have no financial financial relationships relevant to this publication.

Patient consent for publication Not required. 
Ethics approval The study protocol was reviewed and approved by the local Ethics Board.

Provenance and peer review Not commissioned; externally peer reviewed.

Data availability statement Database available if necessary

Open access This is an open access article distributed in accordance with the Creative Commons Attribution Non Commercial (CC BY-NC 4.0) license, which permits others to distribute, remix, adapt, build upon this work non-commercially, and license their derivative works on different terms, provided the original work is properly cited, appropriate credit is given, any changes made indicated, and the use is non-commercial. See: http://creativecommons.org/licenses/by-nc/4.0/.

ORCID iDs

Alejandra Tepox-Padrón http://orcid.org/0000-0002-7812-2003

Adriana Fabiola Romano-Munive http://orcid.org/0000-0002-3835-7465

\section{REFERENCES}

1 Sankaran SJ, Xiao AY, Wu LM, et al. Frequency of progression from acute to chronic pancreatitis and risk factors: a meta-analysis. Gastroenterology 2015;149:1490-500.

2 Guo A, Poneros JM. The role of endotherapy in recurrent acute pancreatitis. Gastrointest Endosc Clin N Am 2018;28:455-76.

3 Forsmark CE, Vege SS, Wilcox CM. Acute pancreatitis. N Engl J Med 2016;375:1972-81.

4 Hammad AY, Ditillo M, Castanon L. Pancreatitis. Surg Clin North Am 2018;98:895-913.

5 Guda NM, Romagnuolo J, Freeman ML. Recurrent and relapsing pancreatitis. Curr Gastroenterol Rep 2011;13:140-9.

$6 \mathrm{Al}-\mathrm{Haddad} \mathrm{M}$, Wallace MB. Diagnostic approach to patients with acute idiopathic and recurrent pancreatitis, what should be done? World J Gastroenterol 2008;14:1007-10.

7 Safari MT, Miri MB, Ebadi S, et al. Comparing the roles of EUS ERCP and MRCP in idiopathic acute recurrent pancreatitis. Clin Med Insights Gastroenterol 2016;9:CGast.S37927-9.

8 Govil A, Agrawal MK, Agrawal D, et al. Role of endoscopic ultrasonography in patients with first episode of idiopathic acute pancreatitis. Indian J Gastroenterol 2014;33:241-8.

9 Somani P, Sunkara T, Sharma M. Role of endoscopic ultrasound in idiopathic pancreatitis. World J Gastroenterol 2017;23:6952-61.

10 Banks PA, Bollen TL, Dervenis C, et al. Classification of acute pancreatitis--2012: revision of the Atlanta classification and definitions by international consensus. Gut 2013;62:102-11.

11 Kadyada SP, Thapa BR, Dhaka N, et al. Role of diagnostic endoscopic ultrasound in idiopathic acute pancreatitis and acute recurrent pancreatitis in children. Pancreas 2019;48:350-5.

12 Kalmin B, Hoffman B, Hawes R, et al. Conventional versus Rosemont endoscopic ultrasound criteria for chronic pancreatitis: comparing interobserver reliability and intertest agreement. Can $J$ Gastroenterol 2011;25:261-4.

13 Dimcevski G, Erchinger FG, Havre R, et al. Ultrasonography in diagnosing chronic pancreatitis: new aspects. World J Gastroenterol 2013;19:7247-57.

14 Stevens T, Parsi MA. Endoscopic ultrasound for the diagnosis of chronic pancreatitis. World J Gastroenterol 2010;16:2841-50.

15 Tandon M, Topazian M. Endoscopic ultrasound in idiopathic acute pancreatitis. Am J Gastroenterol 2001;96:705-9.

16 Shimosegawa T, Chari ST, Frulloni L, et al. International consensus diagnostic criteria for autoimmune pancreatitis: guidelines of the International association of Pancreatology. Pancreas 2011;40:352-8.

$17 \mathrm{Kim} \mathrm{HS}$, Moon JH, Choi HJ, et al. The role of intraductal US in the management of idiopathic recurrent pancreatitis without a definite cause on ERCP. Gastrointest Endosc 2011;73:1148-54.

18 Pereira R, Eslick G, Cox M. Endoscopic ultrasound for routine assessment in idiopathic acute pancreatitis. J Gastrointest Surg 2019;23:1694-700.

19 Morris-Stiff G, Al-Allak A, Frost B, et al. Does endoscopic ultrasound have anything to offer in the diagnosis of idiopathic acute pancreatitis? JOP 2009;10:143-6.

20 Wilcox CM, Varadarajulu S, Eloubeidi M. Role of endoscopic evaluation in idiopathic pancreatitis: a systematic review. Gastrointest Endosc 2006;63:1037-45

21 Yusoff IF, Raymond G, Sahai AV. A prospective comparison of the yield of EUS in primary vs. recurrent idiopathic acute pancreatitis. Gastrointest Endosc 2004;60:673-8.

22 Umans DS, Rangkuti CK, Sperna Weiland CJ, et al. Endoscopic ultrasonography can detect a cause in the majority of patients with idiopathic acute pancreatitis: a systematic review and meta-analysis. Endoscopy 2020;52:955-64.

23 Sajith KG, Chacko A, Dutta AK. Recurrent acute pancreatitis: clinica profile and an approach to diagnosis. Dig Dis Sci 2010;55:3610-6.

24 Vila JJ, Vicuña M, Irisarri R, et al. Diagnostic yield and reliability of endoscopic ultrasonography in patients with idiopathic acute pancreatitis. Scand J Gastroenterol 2010;45:375-81.

25 Guda NM, Trikudanathan G, Freeman ML. Idiopathic recurrent acute pancreatitis. Lancet Gastroenterol Hepatol 2018;3:720-8.

26 Wilcox CM, Seay T, Kim H, et al. Prospective endoscopic ultrasound-based approach to the evaluation of idiopathic pancreatitis: causes, response to therapy, and long-term outcome. Am J Gastroenterol 2016;111:1339-48.

27 Stevens CL, Abbas SM, Watters DAK. How does cholecystectomy influence recurrence of idiopathic acute pancreatitis? J Gastrointest Surg 2016;20:1997-2001.

28 Räty S, Pulkkinen J, Nordback I, et al. Can laparoscopic cholecystectomy prevent recurrent idiopathic acute pancreatitis? a prospective randomized multicenter trial. Ann Surg 2015;262:736-41.

29 Mohan BP. Diagnosis of idiopathic acute pancreatitis: the simpler, the better? Endoscopy 2020;52:965-6. 